\title{
Performance Analysis and Enhancement of TCP in Presence of Jitter in Wireless Networks
}

\author{
Goudru N.G \\ Department of Mathematics, BMS Institute of Technology (affiliated to Visvesvaraya Technological University), \\ Bangalore, India. Mobile: 90-9341384414, \\ E-mail: nggoudru@gmail.com
}

Vijaya Kumar B.P

Department of Information Science and Engineering, MS Ramaiah Institute of Technology (affiliated to Visvesvaraya Technological University), Bangalore, India. Mobile: 90-9980634134,

E-mail: vijaykbp@yahoo.co.in

\begin{abstract}
In wireless networks two types of losses namely congestion loss and transmission loss are significant. One of the important transmission losses is jitter. Variation in inter-arrival time is called jitter. When jitter value is greater than half of the average round trip time cause timeout loss and sender window size falls to one packet resulting reduction in throughput and degradation in the quality of service (QoS). In this paper, we are discussing a new model for transmission control protocol (TCP) which is capable of changing its window size based on the feedback. In the new model TCP is added with intelligence so that it can distinguish the type of losses. If the loss is due to congestion, congestion control algorithm is invoked and loss is due to jitter immediate-recovery algorithm is invoked to recover from the throughput loss. The technique also provides an endto-end congestion control. The performance of TCP is further enhanced by discussing stability. Time-delay control theory is applied for the analysis of asymptotic stability. The stability boundaries of random early detection (RED) control parameter $\mathrm{P}_{\max }$ and jitter control parameter $\beta$ are derived. Using the characteristic equation of Hermite matrix an approximate solution of $q(t)$ (queue length) which converges to a given target value is derived. The results are analyzed based on graphs and statistical data using Matlab R2009b.
\end{abstract}

Index Terms - Congestion, convergence, immediaterecovery, jitter, stability, TCP, wireless networks.

\section{INTRODUCTION}

Because of popularity in Internet services, current network traffic is dominated by the data generated by the applications such as web, email, multimedia downloads, file transfer, etc. [1]. One of the important affecting factors for providing QoS in wireless networks is packet losses. In wireless network, packet losses are due to congestion as well transmission. The congestion loss is due to burst traffic in the network and transmission loss is due to high bit error rate or jitter or frequent disconnections. Transmission loss occurs very frequently in wireless links which cannot be ignored. Packet loss due to transmission in wired links can be ignored [2], [3]. In addition to this, challenges for applying TCP over wireless link is an end-to-end congestion control. An end-to-end congestion control results in seamless data transportation. For a packet sent by the source, acknowledgement (ACK) is received before the timeout period. Otherwise this leads to a timeout loss and sender window (cwnd) size set to 1 packet. In this paper, we consider jitter which causes wireless transmission loss. Jitter is the variation in inter arrival time. Positive jitter value which is greater than half of the average round-trip value results in timeout loss. Any packet arriving after its scheduled time is discarded by the receiver. The problem of delay-jitter is thereby transformed into end-to-end delay and packet losses. Moreover, for a timeout loss, the sender TCP does an exponential backoff for some time. The effect of the backoff is that the window size does not grow for a small period, after which it starts growing at a normal rate. For timeout loss source reduces its sending rate which is unnecessary [4], [5], [6], [7], [20], [21]. Researcher tried to provide some solutions to these problems. Some of the proposed solutions by the researchers are Indirect TCP (I-TCP) Protocol [8], SNOOP protocol [9], Wireless-TCP [10], TCP Westwood [11], ACK pacing [12], ACK splitting [13] and so on. To recognise the loss is due to congestion or transmission, the researchers has proposed some schemes. Some techniques proposed to judge the loss events and improve TCPs throughput are [14], [15]. The problem with these proposed techniques is too difficult in implementation. To satisfy these problems for applying TCP over wireless link, we propose a scheme that can dynamically change the sending rate to the packet loss caused due to congestion or jitter. The model works on the principle of additive increase and multiplicative decrease (AIMD) to satisfy the properties of congestion control algorithm and also has the capability of distinguish the packet loss caused due to congestion or jitter. If the loss is due to congestion, congestion control algorithm is invoked and the loss is due to jitter, 
immediate-recovery algorithm is invoked. This makes sender window to recover from unnecessary reduction made at the source. To enhance the performance of TCP for further, we apply the stability analysis [16], [17], [18]. Conditions are obtained for asymptotic stability of wireless system using time-delay control theory. Using Hermit matrix method, a relationship between $\mathrm{P}_{\max }$ (RED controlling parameter) and $\beta$ (jitter controlling parameter) is established. A stability boundary for $\mathrm{P}_{\max }$ and $\beta$ is established in terms of wireless network resource parameters. Queue convergence analysis is made. An approximate solution for $\mathrm{q}(\mathrm{t})$ is derived. Models validation is made using Matlab. Result analysis is made using graphs and the statistical data.

\section{RATE BASED JITTER ESTIMATION}

The packet delay variation is called jitter. Jitter also can be an indicator of network health. Increasing jitter is an indication of increase in congestion and decreasing jitter is an indication of smoother transmission. This nature of jitter helps to distinguish the congestion loss and the transmission loss [19].

Let $\mathrm{B}_{\mathrm{bi}}$ be the bottleneck bandwidth, $\mathrm{S}$ be the packet size, $t_{0}{ }^{s}$ and $t_{1}{ }^{s}$ be the time when the first and second packets are send one after the other from a source. Let $t_{0}{ }^{d}$ and $\mathrm{t}_{1}{ }^{\mathrm{d}}$ be the time when first and second packets are received at the destination. Without loss of generality, we can write $B_{b n}=S /\left(t_{1}{ }^{d}-t_{0}{ }^{d}\right)$.

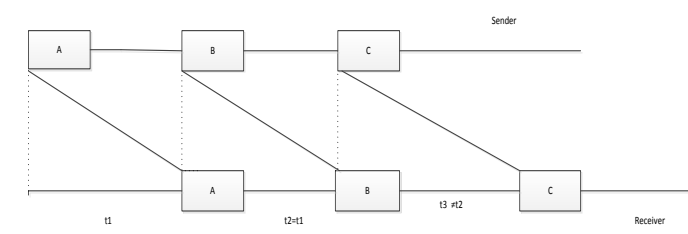

Fig.1. Time Diagram Showing Jitter

Let the receiver receives $i^{\text {th }}$ packet at time $t_{i}{ }^{d}$. But, $t_{i}{ }^{d}=$ $t_{i}^{s}+t t_{i}$, where $t_{i}^{s}$ is the time that $i^{\text {th }}$ packet was sent, and $t_{i}$ is the transmission time. The transmission time $\mathrm{tt}_{\mathrm{i}}=\mathrm{pt}_{\mathrm{i}}$ (propagation delay) $+\mathrm{q}_{\mathrm{i}}$ (queuing delay at the router). In a single hop network where propagation time is same, $\mathrm{pt}_{\mathrm{i}}$ is almost same (constant). The queuing time, $\mathrm{q}_{\mathrm{i}}$ depends on (i) waiting time in the queue, and (ii) processing time which is very negligible. Under these assumptions, the difference between inter-reception time and inter sending time called Jitter. The inter-receipt time between two consecutive packets $i-1$ and $i$ is denoted by irt $_{i}$.

$$
\begin{aligned}
\operatorname{irt}_{\mathrm{i}} & =\mathrm{t}_{\mathrm{i}}^{\mathrm{d}}-\mathrm{t}_{\mathrm{i}-1}{ }^{\mathrm{d}}=\left(\mathrm{t}_{\mathrm{i}}^{\mathrm{s}}+\mathrm{tt}_{\mathrm{i}}\right)-\left(\mathrm{t}_{\mathrm{i}-1}{ }^{\mathrm{s}}+\mathrm{tt}_{\mathrm{i}-1}\right)=\left(\mathrm{t}_{\mathrm{i}}^{\mathrm{s}}-\mathrm{t}_{\mathrm{i}-1}{ }^{\mathrm{s}}\right)+\left(\mathrm{tt}_{\mathrm{i}}\right. \\
\left.-\mathrm{tt}_{\mathrm{i}-1}\right) & \\
\operatorname{irt}_{\mathrm{i}} & =\left(\mathrm{t}_{\mathrm{i}}^{\mathrm{s}}-\mathrm{t}_{\mathrm{i}-1}^{\mathrm{s}}\right)+\left(\left(\mathrm{pt}_{\mathrm{i}}+\mathrm{q}_{\mathrm{i}}\right)-\left(\mathrm{pt}_{\mathrm{i}-1}+\mathrm{q}_{\mathrm{i}-1}\right)\right) \\
\operatorname{irt}_{\mathrm{i}} & =\left(\mathrm{t}_{\mathrm{i}}^{\mathrm{s}}-\mathrm{t}_{\mathrm{i}-1}{ }^{\mathrm{s}}\right)+\left(\mathrm{pt}_{\mathrm{i}}-\mathrm{pt}_{\mathrm{i}-1)}+\left(\mathrm{q}_{\mathrm{i}}-\mathrm{q}_{\mathrm{i}-1}\right)\right.
\end{aligned}
$$

In single hop network propagation time is constant. This implies that $\mathrm{pt}_{\mathrm{i}}$ is constant. Thus

$\left(\mathrm{pt}_{\mathrm{i}}-\mathrm{pt}_{\mathrm{i}-1}\right) \rightarrow 0$. Also, the sending timings of the packets, $t_{i}^{s}$ and $t_{i-1}{ }^{s}$ are known entities. Hence, the only variant is the queuing time. Therefore, jitter is the varying queuing time measured at the destination. Jitter $_{i}=q_{i}-q_{i-1}$. The occurrence of jitter can be noticed by measuring the variation in queuing-delays at the destination. Jitter can be positive, negative, or zero. This leads to the following conclusions. (i) When variation in queuing-delay is positive then $\mathrm{i}^{\text {th }}$ packet gets more delayed than the (i-1) ${ }^{\text {th }}$ packet, (ii) Variation is negative then the $i^{\text {th }}$ packet reach destination in less time than (i-1) th packet, and (iii) variation zero indicates that the network congestion remains unchanged over the time scale of consecutive packets. We are interested in (i). The later packet reaches the destination taking more time than the previous packet, causing positive delay-variation in the inter arrival time. This is due to transmission error and in wireless networks this occurs in more frequent. The positive delay-variation in the inter arrival time results jittering and triggers into timeout loss.

\section{A. K/W Transmission loss-predictor}

Let $L_{t}(t)$ be the wireless transmission loss occurrence after the first packet transferred and ACK has received by the source. Source TCP check whether the jitter-loss is greater than the value of $\mathrm{k} / \mathrm{w}$ or not, where $\mathrm{k}$ is a control parameter and $\mathrm{w}$ is the current window size. The ratio $\mathrm{k} / \mathrm{w}$ indicates that $\mathrm{k}$ packets are in the queue at the router when TCP injects $\mathrm{w}$ packets into the network. Since TCP adds one packet per RTT, $0<\mathrm{k} \leq 1$. Using the scheme proposed in [2], K/W loss-predictor, distinguish the packet losses as follows. (i) The next packet loss is due to congestion when, (a) the source receives triple-duplicate acknowledgement, (b) the arrival of ACK is longer than one RTT (Next-RTT), and Jitter value is greater than $\mathrm{k} / \mathrm{w}$ value. (ii) the next packet loss is due to transmission when, the arrival of ACK is longer than one RTT (NextRTT), and Jitter value is less than or equal to $\mathrm{k} / \mathrm{w}$.

\section{B. Pseudo-code for loss identification}

jitter $_{\mathrm{i}}=\mathrm{q}_{\mathrm{i}}-\mathrm{q}_{\mathrm{i}-1}$ if (next-rtt $>0$ and jitter $r_{i}>\mathrm{k} / \mathrm{w}$ ), \ congestion loss $\beta=0, \backslash$ Invoke congestion control algorithm end

if ( next-rtt $>0$ and jitter $\mathrm{i} \leq \mathrm{k} / \mathrm{w}$ ) $\backslash$ Transmission loss $\beta>0$, Itimeout-loss, invoke immediate-recovery algorithm

end

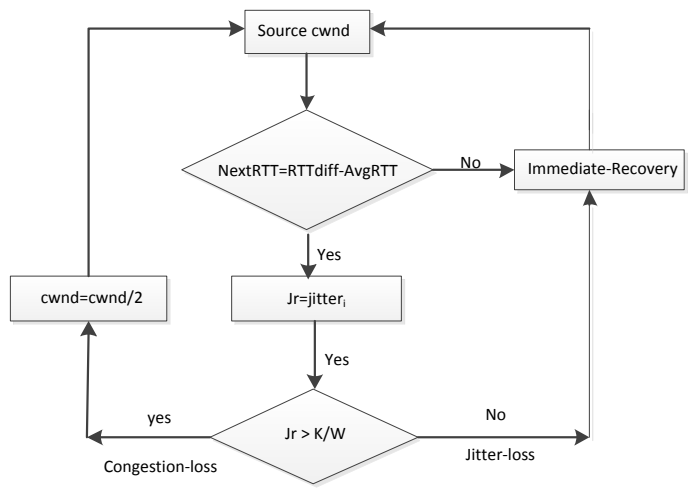

Fig.2. TCP Loss Prediction and Recovery Flow Diagram 
When loss is due to congestion, the jitter control parameter, $\beta=0$. The threshold value of sender window is fixed to half of the current window size, and slow start phase started. When the loss is due to transmission because of jitter, the sender TCP has experienced it as timeout-loss and decreases the window size to 1 which is unnecessary. To recover from this loss the system invoke immediate-recovery module. The recovery rate is proportional to $\beta$ times the flow rate of the previous rtt.

\section{SYSTEM MODEL FOR WIRELESS NETWORKS}

The extended fluid model $[19,20,21]$ that describe the dynamics of the TCP congestion window size in wireless networks in presence of jitter is,

$$
d W(t)=\frac{d t}{R T T}-\alpha W(t) L_{a}(t)+\beta L_{t}(t)
$$

TCP operates on AIMD congestion control strategy. The factor $\alpha$ is decrease rate of source window which is considered as $0.5, \mathrm{~L}_{\mathrm{a}}(\mathrm{t})$ is the arrival rate of packet losses due to congestion at time $\mathrm{t}$ and $\mathrm{L}_{\mathrm{a}}(\mathrm{t})=\frac{\mathrm{w}(\mathrm{t}-\mathrm{R}(\mathrm{t}))}{\mathrm{R}(\mathrm{t}-\mathrm{R}(\mathrm{t}))} \mathrm{p}(\mathrm{t}-$ $\mathrm{R}(\mathrm{t}-\mathrm{R}(\mathrm{t})))$. This loss is proportional to the throughput at the source. First term on the right hand side of equation (1) refers to linear increase of the sender window size until congestion occurs at the destination. The second term refers to congestion control based on the feedback received from the RED router. The third term $\mathrm{L}_{\mathrm{t}}(\mathrm{t})$ refers to immediate-recovery due to jitter loss. The jitter loss is proportional to the sending rate of the source. Therefore, $\mathrm{L}_{\mathrm{t}}(\mathrm{t})$ is proportional to $\frac{\mathrm{w}(\mathrm{t}-\mathrm{R}(\mathrm{t}))}{\mathrm{R}(\mathrm{t}-\mathrm{R}(\mathrm{t}))}$.

The equation (1) can be modified to,

$$
\frac{\partial w}{\partial t}=\frac{1}{R(t)}-\frac{w(t) w(t-R(t))}{2 R(t-R(t))} p(t-R(t))+\beta \frac{w(t-R(t))}{R(t-R(t))}
$$

$\beta$ is jitter loss control parameter and by choice $\beta \in[0,1]$. The dynamic behaviour of instantaneous queue length is given by

$$
\frac{\partial \mathrm{q}(\mathrm{t})}{\partial \mathrm{t}}=\frac{\mathrm{Nw}(\mathrm{t})}{\mathrm{R}(\mathrm{t})}-\mathrm{C}_{\mathrm{d}}
$$

Where, $w(t) / R(t)$ is increase in queue length due to sending rate of the packets by $\mathrm{N}$ - TCP flows. $\mathrm{C}_{\mathrm{d}}=$ $\mathrm{q}(\mathrm{t}) / \mathrm{R}(\mathrm{t})$ is the decrease in queue length due to servicing of packets and delay due to packet departure from the router. The mathematical version which represents packet dropping probability of RED is given by

$$
P(t)=\left\{\begin{array}{cc}
0, & q(t) \in\left[0, t_{\text {min }}\right] \\
\frac{q(t)-t_{\text {min }}}{w_{\text {max }}{ }^{-t}{ }_{\text {min }}} P_{\text {max }}, & q(t) \in\left[t_{\text {min }}, W_{\text {max }}\right] \\
1, & q(t) \geq W_{\text {max }}
\end{array}\right.
$$

Congestion loss is assumed to happen when queue buffer of the router reaches a value of $\mathrm{W}_{\max }$ packets. The maximum buffer size, $\mathrm{W}_{\max }$ of the router is calculated by, $\mathrm{W}_{\text {max }}=\frac{\mathrm{C}_{\mathrm{d}}}{\mathrm{S}} \mathrm{R}(\mathrm{t})+\mathrm{M}$, Where $\mathrm{C}_{\mathrm{d}} / \mathrm{S}$ is the bandwidthdelay product, $\mathrm{M}$ (in packets) is the maximum buffer size of the router and $\mathrm{R}(\mathrm{t})$ is the round trip time. The buffer overflow takes place when the queue length becomes larger than $\mathrm{W}_{\max }$ value. $\mathrm{C}_{\mathrm{d}}$ is the down-link bandwidth. The model describing round trip time (RTT) in wireless networks is given by, $\mathrm{R}(\mathrm{t})=\frac{\mathrm{q}(\mathrm{t})}{\mathrm{C}_{\mathrm{d}}}+\mathrm{T} \mathrm{p}, \mathrm{Tp}$ is the propagation delay in the wireless media, $q(t) / C_{d}$ models the queuing delay.

\section{TIME-DELAY FEEDBACK CONTROL SYSTEM}

In this section, we study the stability analysis of TCP in presence of jitter in wireless network system. The procedure applied is as follows: First linearize the system models, using Hermite matrix for time-delay control system, explicit condition under which the system is asymptotically stability is derived. A relationship for $\mathrm{P}_{\max }$ and $\beta$ is obtained. Mathematical relations are derived for stability boundary of RED control parameter $\mathrm{P}_{\max }$ and jitter control parameter $\beta$ in terms of network resource parameters. Convergence analysis of queue length in presence of jitter is discussed.

\section{A. Linear Model Derivation}

Let $x(t)$ be a general non-linear function defined by, $\mathrm{x}(\mathrm{t})=\mathrm{f}(\mathrm{u}(\mathrm{t}), \mathrm{v}(\mathrm{t}), \mathrm{t})$, where, $\mathrm{u}(\mathrm{t})$ represents the sender window dynamics, and $\mathrm{v}(\mathrm{t})$ represent the queue dynamics at the bottleneck link. We assuming that $f(u(t), v(t), t)$ has smooth and continuous derivatives around the equilibrium point, $\mathrm{Q}_{0}=\left(\mathrm{w}_{0}, \mathrm{R}_{0}, \mathrm{q}_{0}, \mathrm{p}_{0}\right)$. Using Taylor's series expansion, the linear function of nonlinear function, ignoring second and higher order partial derivatives is,

$$
\begin{gathered}
\mathrm{f}(\mathrm{u}(\mathrm{t}), \mathrm{v}(\mathrm{t}), \mathrm{t})=\mathrm{f}\left(\mathrm{u}_{0}(\mathrm{t}), \mathrm{v}_{0}(\mathrm{t}), \mathrm{t}_{0}\right)+\mathrm{f}_{\mathrm{u}}(\mathrm{t}) \delta_{\mathrm{u}}(\mathrm{t}) \\
+\mathrm{f}_{\mathrm{v}}(\mathrm{t}) \delta_{\mathrm{v}}(\mathrm{t})+0\left(\delta_{\mathrm{u}}(\mathrm{t}), \delta_{\mathrm{v}}(\mathrm{t})\right)
\end{gathered}
$$

where $f_{u}(t)=\frac{\partial f}{\partial u}\left|\left(u_{0}, v_{0}\right), f_{v}(t)=\frac{\partial f}{\partial v}\right|\left(u_{0}, v_{0}\right)$. The linear models of the equations (2) to (4) are derived around the equilibrium point $Q_{0}=\left(\mathrm{w}_{0}, \mathrm{R}_{0}, \mathrm{q}_{0}, \mathrm{p}_{0}\right)$. Let $\mathrm{N}$ be the number of TCP flows. At $\mathrm{Q}_{0}$, the steady state conditions of equations (2) and (3) are given by, $\dot{w}(\mathrm{t})=$ $0, \dot{\mathrm{q}}(\mathrm{t})=0$.

The estimation algorithm is based on small signal behaviour dynamics, therefore, at the equilibrium point, without loss of generality, we can assume,

$$
\begin{gathered}
\mathrm{w}(\mathrm{t})=\mathrm{w}(\mathrm{t}-\mathrm{R}(\mathrm{t}))=\mathrm{w}_{0} \\
\mathrm{q}(\mathrm{t})=\mathrm{q}(\mathrm{t}-\mathrm{R}(\mathrm{t}))=\mathrm{q}_{0} \\
\mathrm{p}(\mathrm{t})=\mathrm{p}(\mathrm{t}-\mathrm{R}(\mathrm{t}))=\mathrm{p}_{0} \\
\mathrm{R}(\mathrm{t})=\mathrm{R}(\mathrm{t}-\mathrm{R}(\mathrm{t}))=\mathrm{R}_{0}
\end{gathered}
$$

Using equations (5) in $\dot{\mathrm{w}}(\mathrm{t})=0, \dot{\mathrm{q}}(\mathrm{t})=0$, and after simplification we get, 


$$
\mathrm{p}_{0}=\frac{2 \beta \mathrm{w}_{0}+2}{\mathrm{w}_{0}^{2}}, \mathrm{w}_{0}=\frac{\mathrm{R}_{0} \mathrm{C}_{\mathrm{d}}}{\mathrm{N}}, \mathrm{N}=\frac{\mathrm{R}_{0} \mathrm{C}_{\mathrm{d}}}{\mathrm{w}_{0}}
$$

Let, $\mathrm{w}_{\mathrm{R}}=\mathrm{w}(\mathrm{t}-\mathrm{R}(\mathrm{t})), \mathrm{p}_{\mathrm{R}}=\mathrm{p}(\mathrm{t}-\mathrm{R}(\mathrm{t}))$. From (2) we get,

$$
\begin{aligned}
\mathrm{u}\left(\mathrm{w}, \mathrm{w}_{\mathrm{R}}, \mathrm{q}, \mathrm{p}_{\mathrm{R}}\right)= & \frac{1}{\mathrm{R}(\mathrm{t})}-\frac{\mathrm{w}(\mathrm{t}) \mathrm{w}_{\mathrm{R}}(\mathrm{t})}{2 \mathrm{R}(\mathrm{t})} \mathrm{p}(\mathrm{t}-\mathrm{R}(\mathrm{t})) \\
& +\beta \frac{\mathrm{w}_{\mathrm{R}}(\mathrm{t})}{\mathrm{R}(\mathrm{t})}
\end{aligned}
$$

To linearize equation (6), find all the partial derivatives of $\mathrm{u}\left(\mathrm{w}, \mathrm{w}_{\mathrm{R}}, \mathrm{q}, \mathrm{p}_{\mathrm{R}}\right)$ with respect to the variables at the equilibrium point and defining,

$$
\begin{aligned}
& \delta \mathrm{w}(\mathrm{t})=\mathrm{w}(\mathrm{t})-\mathrm{w}_{0}, \delta \mathrm{q}(\mathrm{t})=\mathrm{q}(\mathrm{t})-\mathrm{q}_{0}, \delta \mathrm{p}(\mathrm{t})=\mathrm{p}(\mathrm{t})- \\
& \mathrm{p}_{0} \\
& \delta \dot{\mathrm{w}}(\mathrm{t})=\dot{\mathrm{w}}(\mathrm{t})-\mathrm{w}_{0}, \delta \dot{\mathrm{q}}(\mathrm{t})=\dot{\mathrm{q}}(\mathrm{t})-\mathrm{q}_{0} \\
& \delta \mathrm{p}(\mathrm{t})=\frac{\mathrm{L}}{\mathrm{B}}\left(\mathrm{q}(\mathrm{t})-\mathrm{t}_{\min }\right)-\mathrm{p}_{0}, \text { where } \mathrm{B}=\mathrm{W}_{\max }-\mathrm{t}_{\min }, \\
& \mathrm{L}=\mathrm{p}_{\max } \\
& \begin{array}{c}
\delta \dot{\mathrm{w}}(\mathrm{t})=-\left(\frac{\beta}{\mathrm{R}_{0}}+\frac{2 \mathrm{~N}}{\mathrm{R}_{0}^{2} \mathrm{C}_{\mathrm{d}}}\right) \delta \mathrm{w}-\frac{\mathrm{C}_{\mathrm{d}}^{2} \mathrm{R}_{0}}{2 \mathrm{~N}^{2}} \delta \mathrm{p}_{\mathrm{R}} \\
\delta \dot{\mathrm{q}}(\mathrm{t})=\frac{\mathrm{N}}{\mathrm{R}_{0}} \delta \mathrm{w}(\mathrm{t})-\frac{1}{\mathrm{R}_{0}} \delta \mathrm{q}(\mathrm{t}) \\
\delta \mathrm{p}\left(\mathrm{t}-\mathrm{R}_{0}\right)=\frac{\mathrm{L}}{\mathrm{B}}\left(\delta \mathrm{q}\left(\mathrm{t}-\mathrm{R}_{0}\right)\right)+\frac{\mathrm{L}}{\mathrm{B}}\left(\mathrm{q}_{0}-\mathrm{t}_{\min }\right)-\mathrm{p}_{0}
\end{array}
\end{aligned}
$$

Using equation (9) in (7), we get

$$
\begin{gathered}
\delta \dot{w}(t)=-\left(\frac{R_{0}}{w_{0}}+\frac{2 N}{R_{0}^{2} C_{d}}\right) \delta w(t)-\frac{L_{d}^{2} R_{0}}{2 N^{2} B} \delta q\left(t-R_{0}\right)+ \\
\frac{L_{d}^{2} R_{0}}{2 N^{2} B}\left(t_{\min }-q_{0}\right)+\frac{R_{0} C_{d}^{2} p_{0}}{2 N^{2}}
\end{gathered}
$$

Denote, $x(t)=\left[\begin{array}{l}\delta w(t) \\ \delta q(t)\end{array}\right]$, then

$$
\dot{x}(t)=A x(t)+E x(t-R(t))+F
$$

Where,

$$
\begin{gathered}
A=\left[\begin{array}{cc}
-\left(\frac{\beta}{\mathrm{R}_{0}}+\frac{2 \mathrm{~N}}{\mathrm{R}_{0}^{2} \mathrm{C}_{\mathrm{d}}}\right) & 0 \\
\frac{\mathrm{N}}{\mathrm{R}_{0}} & \frac{-1}{\mathrm{R}_{0}}
\end{array}\right] \mathrm{E}=\left[\begin{array}{cc}
0 & \frac{-\mathrm{LR}_{0} \mathrm{C}_{\mathrm{d}}^{2}}{2 \mathrm{~N}^{2} \mathrm{~B}} \\
0 & 0
\end{array}\right], \\
F=\left[\begin{array}{c}
\frac{-\mathrm{LR}_{0} \mathrm{C}_{\mathrm{d}}^{2}}{2 \mathrm{~N}^{2} \mathrm{~B}}\left(\mathrm{t}_{\min }-\mathrm{q}_{0}\right)+\frac{\mathrm{R}_{0} \mathrm{C}_{\mathrm{d}} \mathrm{p}_{0}}{2 \mathrm{~N}^{2}} \\
0
\end{array}\right]
\end{gathered}
$$

Solving linear differential equation (11) using Laplace transform technique with

$\mathrm{L}\{\mathrm{x}(\mathrm{t})\}=\mathrm{x}(\mathrm{s})$, we get

$$
\left(\mathrm{sI}-\mathrm{A}-\mathrm{Ee}^{-\mathrm{R}_{0} \mathrm{~s}}\right) \times(\mathrm{s})=\frac{\mathrm{F}}{\mathrm{s}}
$$

$$
\left|s I-A-E e^{-R_{0} s}\right|=0
$$

After simplification, $s^{2}+\left(\frac{\beta}{\mathrm{R}_{0}}+\frac{2 \mathrm{~N}}{\mathrm{R}_{0}^{2} \mathrm{C}_{\mathrm{d}}}+\frac{1}{\mathrm{R}_{0}}\right) \mathrm{s}+$

$$
\left(\frac{\beta}{R_{0}^{2}}+\frac{2 N}{R_{0}^{3} C_{d}}+\frac{L C_{d}^{2}}{2 N B} e^{-R_{0} s}\right)=0
$$

The characteristic equation (14) determines the stability of the closed-loop time-delay wireless system in terms of the state variables $\delta \mathrm{w}(\mathrm{t})$ and $\delta \mathrm{q}(\mathrm{t})$.

\section{B. Stability Analysis}

Denote, $\mathrm{P}\left(\mathrm{s}, \mathrm{e}^{-\mathrm{R}_{0} \mathrm{~s}}\right)=\mathrm{s}^{2}+$

$$
\begin{gathered}
\left(\frac{\beta}{\mathrm{R}_{0}}+\frac{2 \mathrm{~N}}{\mathrm{R}_{0}{ }^{2} \mathrm{C}_{\mathrm{d}}}+\frac{1}{\mathrm{R}_{0}}\right) \mathrm{s}+\left(\frac{\beta}{\mathrm{R}_{0}{ }^{2}}+\frac{2 \mathrm{~N}}{\mathrm{R}_{0}{ }^{3} \mathrm{C}_{\mathrm{d}}}+\frac{\mathrm{LC}_{\mathrm{d}}{ }^{2}}{2 \mathrm{NB}} \mathrm{e}^{-\mathrm{R}_{0} \mathrm{~s}}\right) \\
\text { Let } \mathrm{e}^{-\mathrm{R} 0 \mathrm{~s}}=\mathrm{z} \text {, and } \mathrm{a}_{0}=1, \mathrm{a}_{1}=\frac{\beta}{\mathrm{R}_{0}}+\frac{2 \mathrm{~N}}{\mathrm{R}_{0}{ }^{2} \mathrm{C}_{\mathrm{d}}}+\frac{1}{\mathrm{R}_{0}}, \\
\mathrm{a}_{2}=\frac{\beta}{\mathrm{R}_{0}^{2}}+\frac{2 \mathrm{~N}}{\mathrm{R}_{0}^{3} \mathrm{C}_{\mathrm{d}}}+\frac{\mathrm{LC}_{d}{ }^{2}}{2 \mathrm{NB}} \mathrm{e}^{-\mathrm{R}_{0} \mathrm{~s}} \\
P(\mathrm{~s}, \mathrm{z})=\mathrm{a}_{0} \mathrm{~s}^{2}+\mathrm{a}_{1} \mathrm{~s}+\mathrm{a}_{2}
\end{gathered}
$$

The Hermit matrix for time-delay control system of equation (15) is

$$
H=\left[\begin{array}{ll}
(0,1) & (0,2) \\
(0,2) & (1,2)
\end{array}\right]
$$

$$
\begin{gathered}
(0,1)=2 \mathrm{a}_{0} \mathrm{a}_{1}=\frac{2(\beta+1)}{\mathrm{R}_{0}}+\frac{4 \mathrm{~N}}{\mathrm{R}_{0}{ }^{2} \mathrm{C}_{\mathrm{d}}} \\
(0,2)=-2 \mathrm{a}_{2} \operatorname{Im}(\mathrm{z})=\frac{-\mathrm{Lc}_{d}^{2}}{\mathrm{NB}} \operatorname{Im}(\mathrm{z}) \\
(1,2)=2 \mathrm{a}_{1} \operatorname{Re}\left(\mathrm{a}_{2}\right)= \\
2\left(\frac{\beta}{\mathrm{R}_{0}}+\frac{2 \mathrm{~N}}{\mathrm{R}_{0}^{2} \mathrm{C}_{\mathrm{d}}}+\frac{1}{\mathrm{R}_{0}}\right)\left(\frac{\beta}{\mathrm{R}_{0}{ }^{2}}+\frac{2 \mathrm{~N}}{\mathrm{R}_{0}^{3} \mathrm{C}_{\mathrm{d}}}+\frac{\mathrm{LC}_{\mathrm{d}}^{2}}{2 \mathrm{NB}}\right) \\
\text { Put, } \mathrm{x}_{1}=\frac{\beta}{\mathrm{R}_{0}}+\frac{2 \mathrm{~N}}{\mathrm{R}_{0}{ }^{2} \mathrm{C}_{\mathrm{d}}}+\frac{1}{\mathrm{R}_{0}}, \mathrm{x}_{2}=\frac{\mathrm{LC}_{\mathrm{d}}{ }^{2}}{\mathrm{NB}} \\
\mathrm{x}_{3}=\frac{\beta}{\mathrm{R}_{0}^{2}}+\frac{2 \mathrm{~N}}{\mathrm{R}_{0}^{3} \mathrm{C}_{\mathrm{d}}}
\end{gathered}
$$

Let $z=e^{i \omega}, z=\cos \omega+i \sin \omega, \operatorname{Re}(z)=\cos \omega$, $\operatorname{Im}(z)=\sin \omega$, then

$$
H\left(e^{i \omega}\right)=\left[\begin{array}{cc}
2 x_{1} & -x_{2} \sin \omega \\
-x_{2} \sin \omega & 2 x_{1}\left(x_{3}+\frac{x_{2}}{2} \cos \omega\right)
\end{array}\right]
$$

\section{Stability Conditions}

The time-delayed control system (2) to (4) is asymptotically stable in terms of stable variables $\delta \mathrm{w}(\mathrm{t})$ and $\delta q(\mathrm{t})$, iff the following two conditions are satisfied.

Condition 1: The Hermit matrix $\mathrm{H}(1)=\mathrm{H}\left(\mathrm{e}^{\mathrm{i} 0}\right)$ is positive.

The characteristic equation of (12) is, 


$$
H(1)=\left[\begin{array}{cc}
2 x_{1} & 0 \\
0 & 2 x_{1}\left(x_{3}+\frac{x_{2}}{2}\right)
\end{array}\right]
$$

From the determinant, $4 x_{1}{ }^{2}\left(x_{3}+\frac{x_{2}}{2}\right)>0$, after simplification,

$$
\begin{gathered}
\mathrm{L}=\mathrm{p}_{\max }>-\left(\frac{2 \beta \mathrm{NB}}{\mathrm{R}_{0}{ }^{2} \mathrm{C}_{\mathrm{d}}{ }^{2}}+\frac{4 \mathrm{~N}^{2} \mathrm{~B}}{\mathrm{R}_{0}{ }^{3} \mathrm{C}_{\mathrm{d}}{ }^{3}}\right) \\
\beta>-\left(\frac{\mathrm{p}_{\max } \mathrm{C}_{\mathrm{d}}{ }^{2} \mathrm{R}_{0}{ }^{2}}{2 \mathrm{NB}}+\frac{2 \mathrm{~N}}{\mathrm{R}_{0} \mathrm{C}_{\mathrm{d}}}\right)
\end{gathered}
$$

Condition 2: For all $\omega \in[0,2 \pi]$, det $\mathrm{H}\left(\mathrm{e}^{\mathrm{i} \omega}\right)>0$, leads to the following inequality.

$$
\begin{gathered}
\left(2 x_{1}\right)\left(2 x_{1}\left(x_{3}+\frac{x_{2}}{2}\right)\right) \cos \omega-x_{2}{ }^{2} \sin ^{2} \omega>0 \\
H\left(e^{i \omega}\right)=x_{2}{ }^{2} \cos ^{2} \omega+2 x_{1}{ }^{2} x_{2} \cos \omega+ \\
4 x_{1}{ }^{2} x_{3}-x_{2}{ }^{2}>0
\end{gathered}
$$

The necessary condition for (16) to be true is the discriminate, $\Delta>0$

$$
\cos \omega=\frac{-\mathrm{x}_{1}{ }^{2} \pm \sqrt{\mathrm{x}_{1}{ }^{2}\left(\mathrm{x}_{1}{ }^{2}-4 \mathrm{x}_{3}\right)+\mathrm{x}_{2}{ }^{2}}}{\mathrm{x}_{2}}
$$

By the properties of cosine function, for $\omega \in[0,2 \pi], \cos \omega \in[-1,1]$

The conditions can be written as,

$$
\begin{aligned}
& \frac{-x_{1}{ }^{2}-\sqrt{x_{1}{ }^{2}\left(x_{1}{ }^{2}-4 x_{3}\right)+x_{2}}}{x_{2}}>1
\end{aligned}
$$

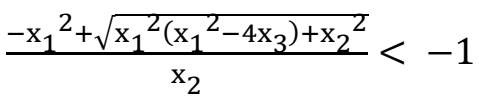

By direct manipulation, there is no solution for the inequality (17). From (18), we obtain

$$
\begin{aligned}
& 0<p_{\max }<\left(\frac{2 \beta N B}{R_{0}^{2} C_{d}^{2}}+\frac{4 N^{2} B}{R_{0}{ }^{3} C_{d}^{3}}\right) \\
& 0<\beta<\left(\frac{\mathrm{p}_{\max } \mathrm{C}_{\mathrm{d}}^{2} \mathrm{R}_{0}^{2}}{2 \mathrm{NB}}+\frac{2 \mathrm{~N}}{\mathrm{R}_{0} \mathrm{C}_{\mathrm{d}}}\right)
\end{aligned}
$$

\section{Theorem 1}

Given the wireless network parameters $\mathrm{C}_{\mathrm{d}}$ (down link capacity), $\mathrm{C}_{\mathrm{u}}$ (up-link capacity), $\mathrm{N}$ (number of TCP sessions), $\mathrm{R}_{0}$ round-trip time, and $\mathrm{B}$ RED control parameter, the wireless network system given by (2) to (4) is asymptotically stable in terms of the state variables $\delta \mathrm{w}(\mathrm{t})$ and $\delta \mathrm{q}(\mathrm{t})$ if and only if the control parameters $\mathrm{P}_{\max }$ and $\beta$ satisfies

$$
0<\mathrm{p}_{\max }<\left(\frac{2 \beta \mathrm{NB}}{\mathrm{R}_{0}^{2} \mathrm{C}_{\mathrm{d}}^{2}}+\frac{4 \mathrm{~N}^{2} \mathrm{~B}}{\mathrm{R}_{0}^{3} \mathrm{C}_{\mathrm{d}}{ }^{3}}\right)
$$

$$
0<\beta<\left(\frac{\mathrm{p}_{\max } \mathrm{C}_{\mathrm{d}}^{2} \mathrm{R}_{0}^{2}}{2 \mathrm{NB}}+\frac{2 \mathrm{~N}}{\mathrm{R}_{0} \mathrm{C}_{\mathrm{d}}}\right)
$$

\section{CONVERgEnce AnAlysis of Dynamic Queue}

In this section, we discuss the convergence of buffer queue in the router. From equation (12),

$$
\left(\mathrm{s} I-\mathrm{A}-\mathrm{E} \mathrm{e}^{-\mathrm{R} 0 \mathrm{~s}}\right)^{-1}=\frac{1}{\mathrm{P}\left(\mathrm{s}, \mathrm{e}^{-\mathrm{R}_{0} \mathrm{~s}}\right)}\left[\begin{array}{ll}
\mathrm{y}_{1} & \mathrm{y}_{2} \\
\mathrm{y}_{3} & \mathrm{y}_{4}
\end{array}\right]
$$

Where, $y_{1}=s+\frac{1}{R_{0}}, y_{2}=-\frac{L R_{0} C_{d}^{2}}{2 N^{2} B} e^{-R_{0} s}, y_{3}=\frac{N}{R_{0}}$, $y_{4}=s+\frac{\beta}{R 0}+\frac{2 N}{R_{0}{ }^{2} C_{d}}$

Equation (13) can be written as

$$
x(s)=\frac{F}{s p\left(s, e^{-R 0 s}\right)}\left[\begin{array}{ll}
y_{1} & y_{2} \\
y_{3} & y_{4}
\end{array}\right]
$$

After simplification, $x(\mathrm{~s})=\frac{\mathrm{y} 5}{\mathrm{sp}\left(\mathrm{e}^{-\mathrm{R}_{0} \mathrm{~s}}\right)}\left[\begin{array}{l}\mathrm{y}_{1} \\ \mathrm{y}_{3}\end{array}\right]$,

$$
\text { or }\left[\begin{array}{l}
\delta \mathrm{W}(\mathrm{s}) \\
\delta \mathrm{Q}(\mathrm{s})
\end{array}\right]=\mathrm{y}_{5}\left[\begin{array}{l}
\mathrm{y}_{1} \\
\mathrm{y}_{3}
\end{array}\right]
$$

Where,

$$
\mathrm{y}_{5}=\frac{\mathrm{LR}_{0} \mathrm{C}_{\mathrm{d}} 2 \frac{\left(\mathrm{t}_{\min }-\mathrm{q}_{0}\right)}{2 \mathrm{~N}^{2} \mathrm{~B}}+\frac{\mathrm{R}_{0} \mathrm{C}_{\mathrm{d}}{ }^{2} \mathrm{p}_{0}}{2 \mathrm{~N}^{2}}}{\mathrm{~s} \mathrm{p}\left(\mathrm{s}, \mathrm{e}^{-\mathrm{R}_{0} \mathrm{~s}}\right)}
$$

From equation (21),

$$
\begin{gathered}
\delta Q(s)=\frac{\frac{\mathrm{N}}{\mathrm{R}_{0}}\left(\frac{\mathrm{LR}_{0} \mathrm{C}_{\mathrm{d}}{ }^{2}}{2 \mathrm{~N}^{2} \mathrm{~B}}\left(\mathrm{t}_{\min }-\mathrm{q}_{0}\right)+\frac{\mathrm{R}_{0} \mathrm{C}_{\mathrm{d}}{ }^{2} \mathrm{p}_{0}}{2 \mathrm{~N}^{2}}\right)}{\mathrm{sP}\left(\mathrm{s}, \mathrm{e}^{-\mathrm{R}_{0} \mathrm{~s}}\right)} \\
\delta \mathrm{Q}(\mathrm{s})=\frac{\left(\frac{\mathrm{C}_{\mathrm{d}}{ }^{2}}{2 \mathrm{~N}}\right)\left(\frac{\mathrm{L}}{\mathrm{B}}\left(\mathrm{t}_{\min }-\mathrm{q}_{0}\right)+\mathrm{p}_{0}\right)}{\mathrm{sp}\left(\mathrm{s}, \mathrm{e}^{-\mathrm{R}_{0} \mathrm{~s}}\right)}
\end{gathered}
$$

$$
\begin{gathered}
\delta Q(s)=\frac{\frac{1 \mathrm{C}_{\mathrm{d}}{ }^{2}}{\mathrm{~s} 2 \mathrm{~N}}\left(\frac{\mathrm{L}}{\mathrm{B}}\left(\mathrm{t}_{\min ^{-}}-\mathrm{q}_{0}\right)+\mathrm{p}_{0}\right)}{\mathrm{s}^{2}+\left(\frac{\beta}{\mathrm{R}_{0}}+\frac{2 \mathrm{~N}}{\mathrm{R}_{0}{ }^{2} \mathrm{C}_{\mathrm{d}}}+\frac{1}{\mathrm{Ro}}\right) \mathrm{s}+\left(\frac{\beta}{\mathrm{R}_{0}} 2+\frac{2 \mathrm{~N}}{\mathrm{R}_{0}{ }^{3} \mathrm{C}_{\mathrm{d}}}\right)+\left(\frac{\mathrm{LC}_{\mathrm{d}}}{2 \mathrm{~N} \mathrm{~B}}\right) \mathrm{e}^{-\mathrm{R}_{0} \mathrm{~s}}} \\
\delta \mathrm{Q}(\mathrm{s})=\frac{\mathrm{y} 6}{\mathrm{~s}^{2}+\mathrm{x}_{1} \mathrm{~s}+\mathrm{x}_{3}+\frac{\mathrm{x}_{2}}{2} \mathrm{e}^{-\mathrm{R}_{0} \mathrm{~s}}}
\end{gathered}
$$

Where,

$$
\mathrm{y}^{6}=\frac{1}{\mathrm{~s}} \frac{\mathrm{C}_{\mathrm{d}}^{2}}{2 \mathrm{~N}}\left(\frac{\mathrm{L}}{\mathrm{B}}\left(\mathrm{t}_{\min }-\mathrm{q}_{0}\right)+\mathrm{p}_{0}\right)
$$

\section{A. Theorem 2}

Given the wireless network parameters $C_{d}, C_{u}, N, R_{0}$ and $\mathrm{B}$, on the basis of wireless network system given in (2) to (4), an approximate solution of $q(t)$ is

$$
q(t)=q_{0}+y_{6}\left[\frac{e^{x t}}{(x-y) x}-\frac{e^{y t}}{(x-y) y}+\frac{1}{x y}\right]
$$


If the RED control parameter $\mathrm{P}_{\max }$ and jitter control parameter $\beta$ satisfies

$$
\begin{gathered}
0<p_{\max } \leq \frac{-\xi_{2}+\sqrt{\xi_{2}{ }^{2}-4 \xi_{1} \xi_{3}}}{2 \xi_{1}} \\
0<\beta \leq \frac{-\mathrm{A}_{2}+\sqrt{\mathrm{A}_{2}{ }^{2}-4 \mathrm{~A}_{1} \mathrm{~A}_{3}}}{2 \mathrm{~A}_{1}}
\end{gathered}
$$

Where $\xi_{i}$ 's and $A_{i}$ 's are given in (28).

Proof: Expanding $\mathrm{e}_{0}^{-\mathrm{R} s}$, using Taylor's theorem about the stability point discarding the terms of order three and higher,

$$
\mathrm{e}^{-\mathrm{R}_{0} \mathrm{~s}}=1-\mathrm{R}_{0} \mathrm{~s}+\frac{\mathrm{R}_{0}{ }^{2} \mathrm{~s}^{2}}{2}
$$

From (23), $s^{2}+\mathrm{x}_{1} \mathrm{~s}+\mathrm{x}_{3}+\frac{\mathrm{x}_{2}}{2} \mathrm{e}^{-\mathrm{R}_{0} \mathrm{~s}}$.

This simplifies to

$$
\begin{gathered}
\mathrm{s}^{2}+\left(\frac{\beta}{\mathrm{R}_{0}}+\frac{2 \mathrm{~N}}{\mathrm{R}_{0}^{2} \mathrm{C}_{\mathrm{d}}}+\frac{1}{\mathrm{R}_{0}}\right) \mathrm{s}+ \\
\left(\frac{\beta}{\mathrm{R}_{0}^{2}}+\frac{2 \mathrm{~N}}{\mathrm{R}_{0}^{3} \mathrm{C}_{\mathrm{d}}}+\frac{\mathrm{LC}_{d}^{2}}{2 \mathrm{NB}}\left(1-\mathrm{R}_{0}+\frac{\mathrm{R}_{0}^{2} \mathrm{~s}^{2}}{2}\right)\right) \\
\left(1+\frac{\mathrm{LR}_{0}^{2} \mathrm{C}_{\mathrm{d}}^{2}}{4 \mathrm{NB}}\right) \mathrm{s}^{2}+\left(\frac{\beta}{\mathrm{R}_{0}}+\frac{2 \mathrm{~N}}{\mathrm{R}_{0}^{2} \mathrm{C}_{\mathrm{d}}}+\frac{1}{\mathrm{R}_{0}}-\frac{\mathrm{LR}_{0} \mathrm{C}_{\mathrm{d}}{ }^{2}}{2 \mathrm{NB}}\right) \mathrm{s} \\
+\left(\frac{\beta}{\mathrm{R}_{0}^{2}}+\frac{2 \mathrm{~N}}{\mathrm{R}_{0}^{3} \mathrm{C}_{\mathrm{d}}}+\frac{\mathrm{LC}_{\mathrm{d}}{ }^{2}}{2 \mathrm{NB}}\right)
\end{gathered}
$$

Put

$$
\begin{gathered}
\eta_{1}=\left(1+\frac{\mathrm{LR}_{0}^{2} \mathrm{C}_{\mathrm{d}}^{2}}{4 \mathrm{NB}}\right), \eta_{2}=\frac{\beta}{\mathrm{R}_{0}}+\frac{2 \mathrm{~N}}{\mathrm{R}_{0}{ }^{2} \mathrm{C}_{\mathrm{d}}}+\frac{1}{\mathrm{R}_{0}}-\frac{\mathrm{LR}_{0} \mathrm{C}_{\mathrm{d}}^{2}}{2 \mathrm{NB}} \\
\eta_{3}=\frac{\beta}{\mathrm{R}_{0}{ }^{2}}+\frac{2 \mathrm{~N}}{\mathrm{R}_{0}^{3} \mathrm{C}_{\mathrm{d}}}+\frac{\mathrm{LC}_{\mathrm{d}}{ }^{2}}{2 \mathrm{NB}} \\
\eta_{1} \mathrm{~s}^{2}+\eta_{2} \mathrm{~s}+\eta_{3}
\end{gathered}
$$

If $\Delta>0$, equation (24) has distinct roots given by

$$
x, y=\frac{-\eta_{2} \pm \sqrt{\eta_{2}^{2}-4 \eta_{1} \eta_{3}}}{2 \eta_{1}}
$$

where, $\mathrm{x}$ takes + and $\mathrm{y}$ takes - of \pm .

The discriminate of (25) is positive. After simplification for the discriminant, and expressing in terms of $L$ and $\beta$, we get,

$$
\begin{aligned}
& \xi_{1} L^{2}+\xi_{2} L+\xi_{3}>0 \\
& A_{1} \beta^{2}+A_{2} \beta+A_{3}>0
\end{aligned}
$$

where,

$$
\begin{gathered}
\xi_{1}=\left(\frac{C_{d}^{2} R_{0}}{2 N B}\right)^{2} \\
\xi_{2}=\frac{2 \beta c_{d}^{2}}{N B}+\frac{4 C_{d}}{R_{0} B}+\frac{C_{d}^{2}}{N B} \\
\xi_{3}=\frac{4 \beta}{R_{0}{ }^{2}}-\left(\frac{\beta}{R_{0}}+\frac{1}{R_{0}}-\frac{2 N}{R_{0}{ }^{2} C_{d}}\right)^{2}
\end{gathered}
$$

$$
\begin{gathered}
\mathrm{A}_{1}=\frac{1}{\mathrm{R}_{0}{ }^{2}} \\
\mathrm{~A}_{2}=\frac{4 \mathrm{~N}}{\mathrm{R}_{0}^{3} \mathrm{C}_{\mathrm{d}}}-\frac{2}{\mathrm{R}_{0}{ }^{2}}-\frac{2 \mathrm{LC}_{\mathrm{d}}{ }^{2}}{\mathrm{NB}} \\
\mathrm{A}_{3}=\frac{1}{\mathrm{R}_{0}}+\frac{4 \mathrm{~N}^{2}}{\mathrm{R}_{0}{ }^{4} \mathrm{C}_{\mathrm{d}}{ }^{2}}-\frac{4 \mathrm{~N}}{\mathrm{R}_{0}{ }^{3} \mathrm{C}_{\mathrm{d}}}- \\
\left(\frac{\mathrm{LC}_{\mathrm{d}}{ }^{2} \mathrm{R}_{0}}{2 \mathrm{NB}}\right)^{2}-\frac{\mathrm{LC}_{\mathrm{d}}{ }^{2}}{\mathrm{NB}}-\frac{4 \mathrm{LC}_{\mathrm{d}}}{\mathrm{R}_{0} \mathrm{~B}}
\end{gathered}
$$

Solving (26) for $\mathrm{L}=\mathrm{P}_{\max }$ and (27) for $\beta$, we get

$$
\begin{gathered}
0<p_{\max } \leq \frac{-\xi_{2}+\sqrt{\xi_{2}{ }^{2}-4 \xi_{1} \xi_{3}}}{2 \xi_{1}} \\
0<\beta \leq \frac{-\mathrm{A}_{2}+\sqrt{\mathrm{A}_{2}{ }^{2}-4 \mathrm{~A}_{1} \mathrm{~A}_{3}}}{2 \mathrm{~A}_{1}}
\end{gathered}
$$

From Equation (23)

$$
\begin{gathered}
\delta Q(s)=\frac{y_{6}}{s\left(\eta_{1} s^{2}+\eta_{2} s+\eta_{3}\right)} \\
\delta Q(s)=\frac{y_{6}}{s(s-x)(s-y)}
\end{gathered}
$$

Using partial fraction,

$$
\delta Q(s)=y_{6}\left[\frac{1}{x(x-y)} \frac{1}{(s-x)}-\frac{1}{y(x-y)} \frac{1}{s-y}+\frac{1}{x y} \frac{1}{s}\right]
$$

Taking Laplace transform,

$$
\begin{gathered}
\delta q(t)=y_{6}\left[\frac{e^{x t}}{x(x-y)}-\frac{e^{y t}}{y(x-y)}+\frac{1}{x y}\right] \\
q(t)=q_{0}+y_{6}\left[\frac{e^{x t}}{x(x-y)}-\frac{e^{y t}}{y(x-y)}+\frac{1}{x y}\right]
\end{gathered}
$$

\section{B. Theorem 3}

Given the wireless network parameters $C_{d}, C_{u}, N, R_{0}$ and $\mathrm{B}$, the instantaneous queue length converges to the target, $T=\frac{\left(t_{\min }+w_{\max }\right)}{2}$ if and only if RED control parameter, $\mathrm{P}_{\max }$ and jitter control parameter $\beta$ satisfies

$$
\begin{gathered}
\mathrm{P}_{\text {max }}=\mathrm{K}_{1} \mathrm{~K}_{2}+\mathrm{K}_{3} \\
\beta=\mathrm{K}_{4} \mathrm{~K}_{5}
\end{gathered}
$$

where Ki's are given by (35).

Proof: From equation (23),

$$
\begin{gathered}
\lim _{t \rightarrow \infty} \delta q(t)=\lim _{s \rightarrow 0} s Q(s)= \\
\lim _{s \rightarrow 0} \frac{v 6}{s^{2}+x_{1} s+x_{3}+\frac{x_{2}}{2} e^{-R_{0} s}} \\
\lim _{t \rightarrow \infty} \delta q(t)=\lim _{s \rightarrow 0} s Q(s)=\frac{y_{6}}{x_{3}+x_{2} / 2}+q_{0} \\
\lim _{t \rightarrow \infty} q(t)=q_{0}+\lim _{t \rightarrow \infty} \delta q(t)
\end{gathered}
$$




$$
\begin{aligned}
\lim _{t \rightarrow \infty} q(t) & =q_{0}+\frac{y 6}{x 3+\frac{x 2}{2}} \\
\text { Let } \lim _{t \rightarrow \infty} q(t) & =\frac{t_{\text {min }}+w_{\max }}{2}
\end{aligned}
$$

Then

$$
\begin{gathered}
\mathrm{P}_{\text {max }}=\mathrm{K}_{1} \mathrm{~K}_{2}+\mathrm{K}_{3} \\
\beta=\mathrm{K}_{4} \mathrm{~K}_{5}
\end{gathered}
$$

Where

$$
\begin{gathered}
\mathrm{K}_{1}=\frac{4 \mathrm{~N}^{2}}{\mathrm{R}_{0}{ }^{3} \mathrm{C}_{\mathrm{d}}{ }^{3}}+\frac{2 \beta \mathrm{N}}{\mathrm{R}_{0}{ }^{2} \mathrm{C}_{\mathrm{d}}{ }^{2}} \\
\mathrm{~K}_{2}=2 \mathrm{q}_{0}-\mathrm{t}_{\min }-\mathrm{w}_{\max } \\
\mathrm{K}_{3}=\frac{4 \beta \mathrm{N}}{\mathrm{R}_{0} \mathrm{C}_{\mathrm{d}}}+\frac{4 \mathrm{~N}^{2}}{\mathrm{R}_{0}{ }^{2} \mathrm{C}_{\mathrm{d}}{ }^{2}} \\
\mathrm{~K}_{4}=\frac{\mathrm{P}_{\max } \mathrm{R}_{0}{ }^{2} \mathrm{C}_{\mathrm{d}}{ }^{2}-4 \mathrm{~N}^{2}}{2 \mathrm{~N}+4 \mathrm{NR}_{0} \mathrm{C}_{\mathrm{d}}} \\
\mathrm{K}_{5}=1+\frac{1}{\mathrm{R}_{0} \mathrm{C}_{\mathrm{d}}}\left(2 \mathrm{q}_{0}-\mathrm{t}_{\min }-\mathrm{w}_{\text {max }}\right)
\end{gathered}
$$

Theorem 3 gives a relationship between $\mathrm{P}_{\max }, \beta$ and $\mathrm{q}_{0}$ The relations given in (33), (34) and (35) not only leads to the stability of the system but also brings the instantaneous queue length to converge to the given target value.

\section{Theorem 4}

The wireless network system (2) to (4) is asymptotically stable in terms of the state variable $\delta \mathrm{w}(\mathrm{t})$, $\delta q(t)$, if the equilibrium value of the queue level $q_{0}$ satisfies

$$
\begin{gathered}
\frac{\mathrm{t}_{\min }+\mathrm{w}_{\max }}{2}-\frac{\mathrm{R}_{0} \mathrm{C}_{\mathrm{d}}}{2}<\mathrm{q}_{0} \leq \\
\frac{\mathrm{t}_{\min }+\mathrm{w}_{\max }}{2}-\frac{\mathrm{R}_{0} \mathrm{C}_{\mathrm{d}}}{2}-\frac{\xi_{2}}{\xi_{7}}+\frac{\xi_{8}}{\xi_{7}} \sqrt{\xi_{6}}
\end{gathered}
$$

Proof: Using (33) in (29)

$$
0<\mathrm{K}_{1} \mathrm{~K}_{2}+\mathrm{K}_{3} \leq \frac{-\xi_{2}+\sqrt{\xi_{2}^{2}-4 \xi_{1} \xi_{3}}}{2 \xi_{1}}
$$

Substituting for K1, K2 and K3 from (35), and simplifying for $\mathrm{q}_{0}$, we get

$$
\begin{gathered}
\frac{\mathrm{t}_{\min }+\mathrm{w}_{\max }}{2}-\frac{\mathrm{R}_{0} \mathrm{C}_{\mathrm{d}}}{2}<\mathrm{q}_{0} \leq \\
\frac{\mathrm{t}_{\min }+\mathrm{w}_{\max }}{2}-\frac{\mathrm{R}_{0} \mathrm{C}_{\mathrm{d}}}{2}-\frac{\xi_{2}}{\xi_{7}}+\frac{\xi_{8}}{\xi_{7}} \sqrt{\xi_{6}} \\
\xi_{6}=\frac{\mathrm{C}_{d}{ }^{2}}{\mathrm{~N}^{2} \mathrm{~B}^{2}}\left[\left(2 \beta+1+\frac{4 \mathrm{~N}}{\mathrm{R}_{0} \mathrm{C}_{\mathrm{d}}}\right)^{2}+\right. \\
\left.(\beta-1)^{2}+\frac{4 \mathrm{~N}}{\mathrm{R}_{0} \mathrm{C}_{\mathrm{d}}}\left(\frac{\mathrm{N}}{\mathrm{R}_{0} \mathrm{C}_{\mathrm{d}}}-\beta-1\right)\right]
\end{gathered}
$$

$$
\begin{gathered}
\xi_{7}=\frac{2 C_{d}^{3}}{N^{2}}\left(\beta+\frac{2 N}{R_{0} C_{d}}\right) \\
\xi_{8}=\frac{C_{d}^{2}}{N B}
\end{gathered}
$$

Theorem 4 gives a stable value for $\mathrm{q}_{0}$. With help of this value one can tune the network system parameters to achieve queue convergence and hence obtain good throughput.

\section{SimUlation AND PERFORMANCE ANALYSIS}

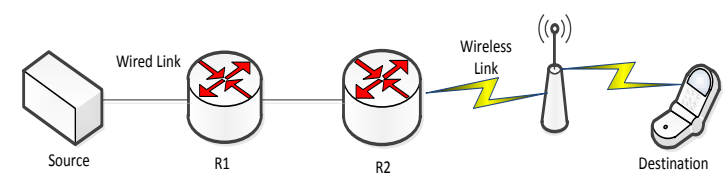

Fig.3. Network Model

A number of simulation experiments are conducted using Matlab R2009b to evaluate the performance of TCP. Fig.3 shows a typical network topology of a heterogeneous network. $\mathrm{R}_{1}$ and $\mathrm{R}_{2}$ are the routers and the TCP which has been modeled represents the last hop transmission between $R_{2}$ and destination. The propagation time is assumed to be $50 \mathrm{~ms}$. The bottleneck bandwidth $\left(\mathrm{C}_{\mathrm{d}}\right)$ is $10 \mathrm{Mbps}$, packet size is 1000 bytes and load factor is $10 \mathrm{TCP}$ sessions, $\mathrm{k}=1, \mathrm{R}_{0}=0.1 \mathrm{~s}$.

A. Experiment1: Un-stable network system

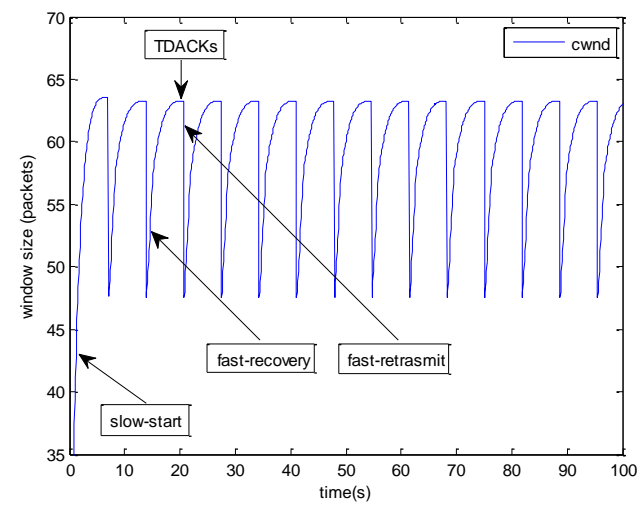

Fig.4. Slow-Start, Congestion Control and Recovery

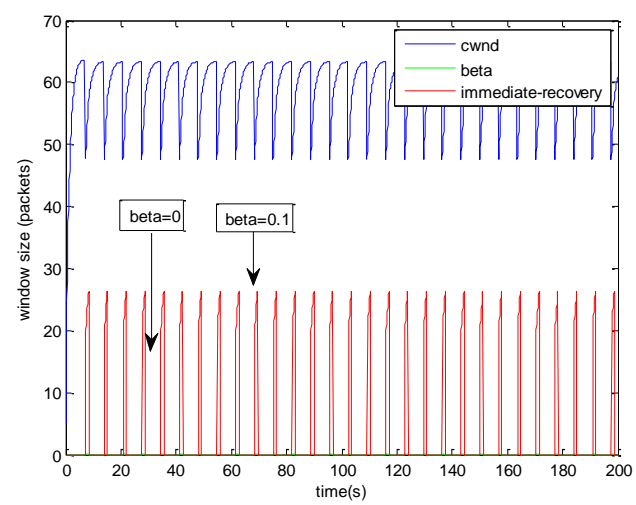

Fig.5. Cwnd Recovery due to Jitter-Loss 

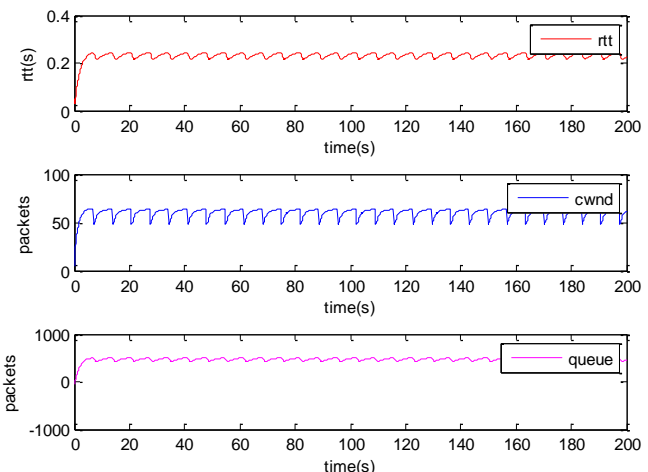

Fig.6. Performance of rtt, cwnd and Queue w.r.t Time

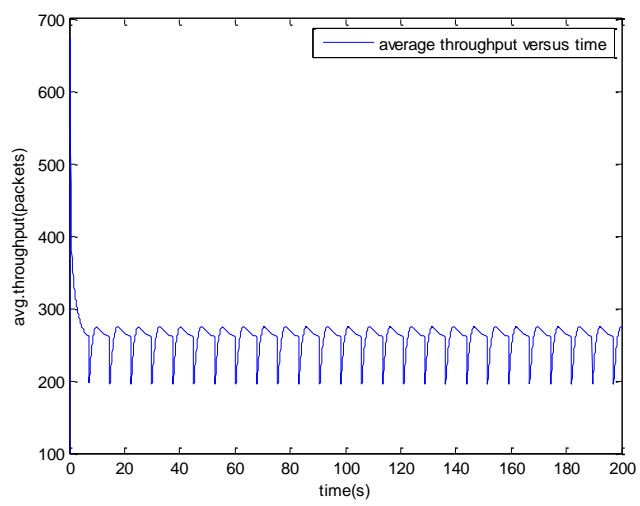

Fig.7. Performance of Throughput w.r.t Time

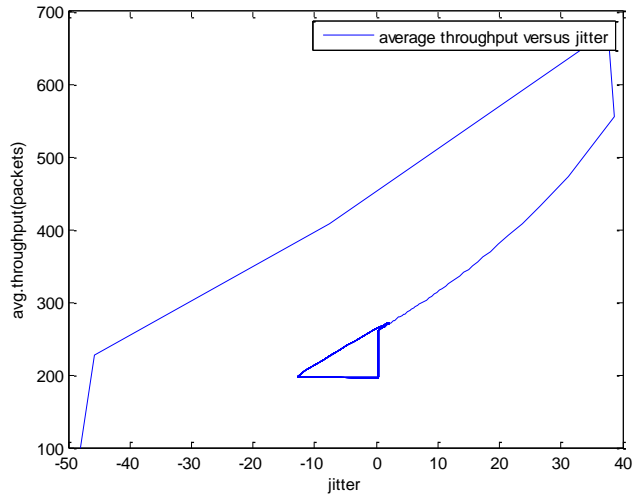

Fig.8. Variation of Throughput w.r.t Jitter

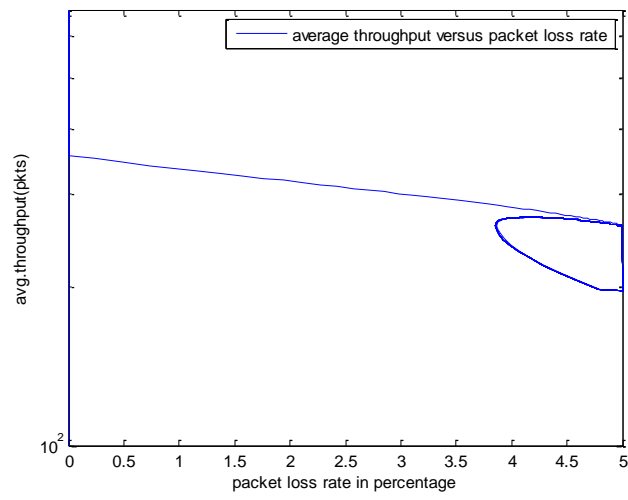

Fig.9. Performance of Throughput w.r.t pkt. Loss
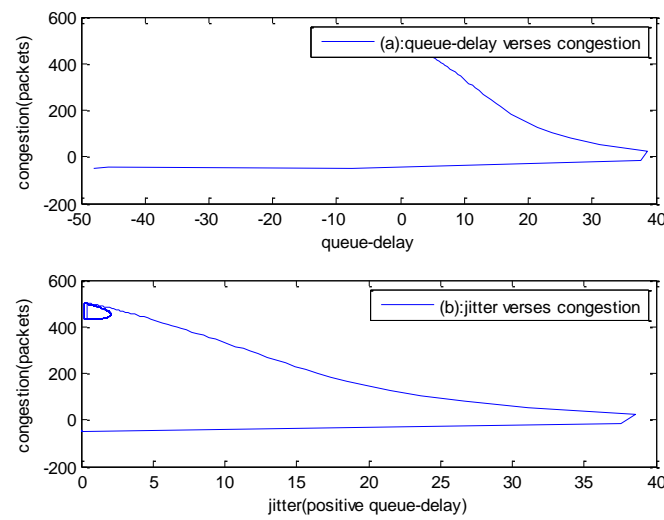

Fig.10. (a) Congestion due to Queue-Delay (b) Congestion due to Jitter

The trace of the graph of fig.4 represents the typical behaviour of cwnd. The slow-start phase initiate data flow over the connection and increases continuously for every rtt until loss occurs. When the sender cwnd reaches a maximum size of 64 packets, buffer in the bottleneck router starts overflowing. The source receives TDAKs, the cwnd is reduced to half of its current value. For a timeout loss the window size reduces to 1 packet. In addition to timeout loss, the sender does an exponential backoff for some time. The effect of backoff is that the window size does not grow for a small period, after which it starts growing in the normal rate.

To add intelligence to TCP that packet loss is due to transmission and not due to congestion, and mitigate backoff loss, we introduce the term $\mathrm{L}_{\mathrm{t}}(\mathrm{t})$ in equation (2). The traces in fig.5 represent immediate-recovery of sender window due to jittering. The timeout loss experienced by the source and reduction made in its sending rate is unnecessary. To recover the loss TCP initiates immediate-recovery algorithm. The amount of recovery is proportional to sending rate in the previous RTT. The jitter value is compared with $\mathrm{k} / \mathrm{w}$ value. If it is congestion loss then $\beta=0$, congestion control algorithm is invoked. If it is jitter loss then $\beta=0.1$. The traces of fig. 6 represent that the queue length oscillates with the sender window and similarly RTT varies with queue length. As the sender window increases, the queue length also increase because the sending rate by the source is higher than the service rate by the bottleneck router. The bottleneck bandwidth considered is $10 \mathrm{Mb} / \mathrm{s}$. When the cwnd reaches 44 packets then the queue length reaches a minimum threshold value of 200 packets. We observe that when the cwnd is between 45-64 packets, number of packets in the queue is higher than 200 and the congestion avoidance event starts. The bottleneck router stars dropping the packets randomly. Sender TCP on receiving TDACKs, halves its cwnd size.

Throughput depends on the source sending rate. The graph of fig.7 shows that throughput varies over the range of 200 to 280 packets corresponding to cwnd 47 and 64 packets respectively. The traces of fig. 8 show the behaviour of throughput with respect to jitter. In the beginning of the simulation, jitter value is negative resulting highest throughput. As the simulation time lapses jitter value becomes positive resulting in the 
decrease of throughput. When the jitter value reaches a maximum of $40 \mathrm{~ms}$ throughput drops suddenly to a small value. Traces of fig. 9 represent that throughput decreases with the increase of percentage packet loss rate. When the loss rate is between $4 \%$ to $5 \%$, throughput falls by $30 \%$ but recovers immediately. The occurrence of jitter can be noticed by measuring the variation in queuing-delay at the destination. fig. 10(a) represents the variation of congestion in the bottleneck router with respect to (w.r.t) queue-delay. The later packet reaches the destination taking more time than the previous packet, causing variation in the inter arrival time of packets. This is due to transmission and is more frequent in wireless networks. Thus, Jittering triggers into timeout loss. fig. 10(b) gives the traces of congestion with respect to jitter. From the graph, the congestion slowly builds up with respect to increase in jitter, when jitter decrease, congestion reaches a maximum value of 500 packets because of the fact that many TCPs sessions are pumping large number of packet into the network (load), resources are being shared optimally.

\section{B. Experiment 2: Stable network system}
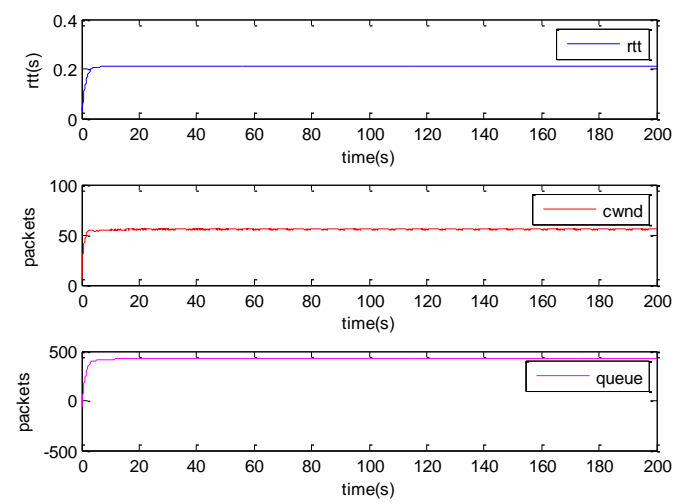

Fig.11. Asymptotic Stability of rtt, cwnd, Queue

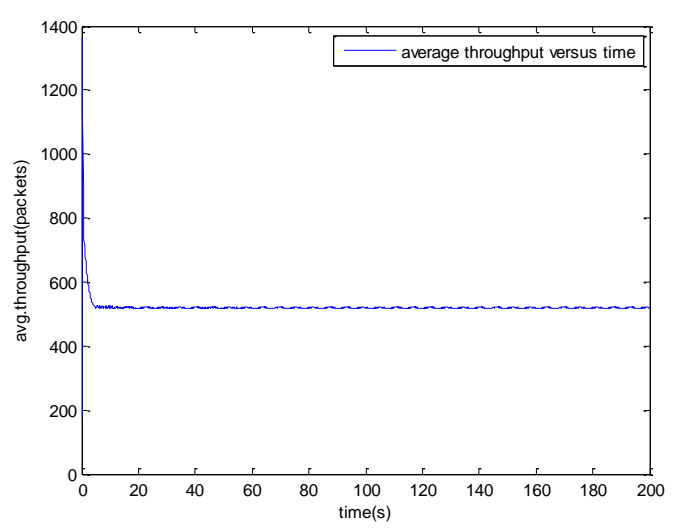

Fig.12. Stable Throughput

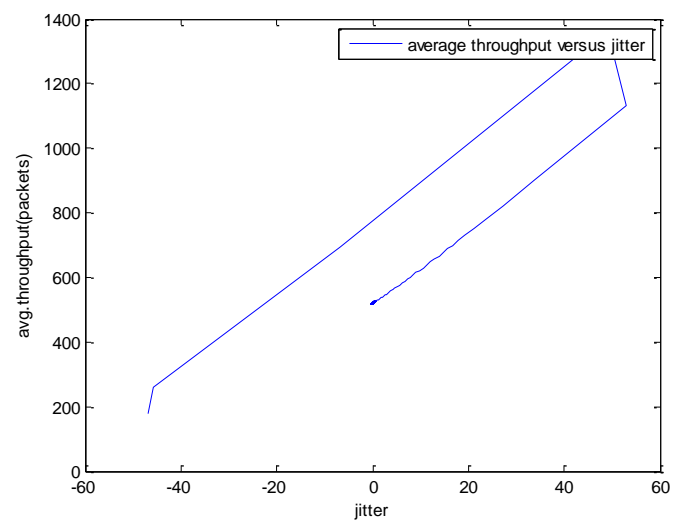

Fig.13. Stable Throughput w.r.t Jitter

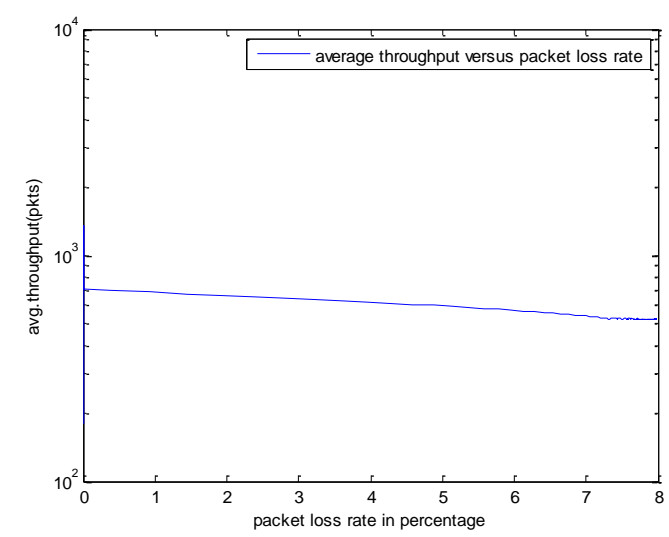

Fig.14. Stable Throughput w.r.t pkt-loss
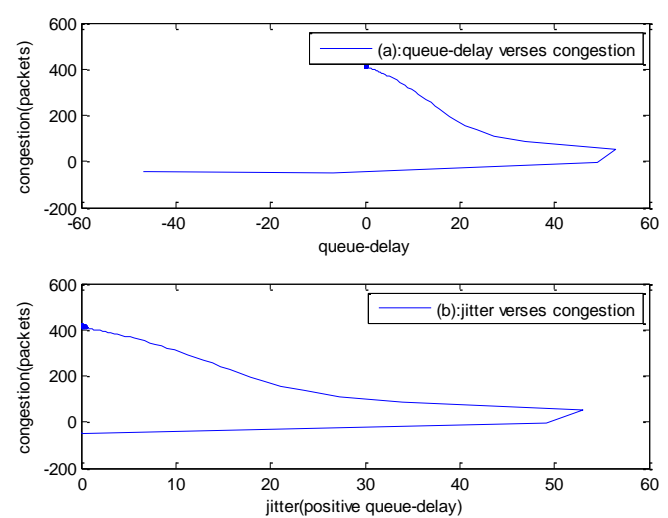

Fig.15. (a) Stable Congestion due to Queue-Delay (b) Stable Congestion due to Jitter

Fig. 11 to fig. 15 are the graphs of stable network system. The experiment was conducted by assuming the propagation delay as $50 \mathrm{~ms}$. The bottleneck bandwidth is $10 \mathrm{Mbps}$, packet size is 1000 bytes and load factor is 10 TCP sessions. Minimum and maximum threshold values of the queue buffer are 200 and 500 packets respectively. The initial values of $R_{0}=0.04 \mathrm{~s}$ and $\beta=0$ and using relation (19), initial value of $p_{\max }$ was calculated. Using this initial $\mathrm{p}_{\max }$ value and relation (20), next value of $\beta$ was calculated. After small laps of simulation time, $\mathrm{P}_{\text {max }}$ and $\beta$ converges to values 0.1067 and 0.3846 respectively. Traces of fig. 10 and fig. 11 shows that rtt, sender 
window size, queue length and throughput values stabilise to $0.21 \mathrm{~s}, 56$ packets, 425 packets and 520 packets respectively. The graph of fig. 13, fig.14 and fig. 15 gives the variation of throughput with respect to jitter, variation of throughput with respect to packet-loss rate and variation of congestion with respect to jitter respectively. The graphs are smooth and not oscillatory. The experimental results shows that for a given resources, stability boundary increases the throughput value by $30 \%$ by minimising the packet losses.

\section{Experiment 3: Queue convergence analysis}

The simulation was conducted for different target values as summarised in table 1. For each of the simulations, the stability range of $\mathrm{q}_{0}$ was calculated using relation (36), Using the $q_{0}$ value, $P_{\max }$ value was calculated using relation (29) with an initial value of $\beta=0.1$, and finally using the value of $p_{\max }, \beta$ was estimated using relation (30). In simulation 1 , target queue length, $\mathrm{T}=500$, the range of $\mathrm{q}_{0}$ is $(459.8,464.96)$, chose $\mathrm{q} 0=462.38$, Value of $\mathrm{P}_{\max }=0.544$, and $\beta=0.0089$. The graph of instantaneous queue length is as illustrated in fig. 4.16. Approximately, $93 \%$ of the traces of the graph values fall around $\mathrm{q}_{0}$. In simulation 2 , target queue length, $\mathrm{T}=550$, the range of $\mathrm{q}_{0}$ is $(506.32,512.69)$, chose $\mathrm{q}_{0}=509.5$, Value of $\mathrm{P}_{\max }=0.0013$, and $\beta=0.0024$. The graph of instantaneous queue length is as illustrated in fig. 17. About $96 \%$ of the traces of the graph values fall around $\mathrm{q}_{0}$. In simulation 3 , target queue length, $\mathrm{T}=600$, the range of $\mathrm{q}_{0}$ is $(555.16,561.03)$, chose $\mathrm{q} 0=558.09$, Value of $\mathrm{P}_{\max }=0.112$, and $\beta=0.0018$. The graph of instantaneous queue length is as illustrated in fig. 18 . Almost, $93 \%$ of the traces of the graph values fall around $\mathrm{q}_{0}$.

Table 1. Simulation results

\begin{tabular}{|c|c|c|c|c|c|c|}
\hline $\mathbf{1}$ & $\mathbf{2}$ & $\mathbf{3}$ & $\mathbf{4}$ & $\mathbf{5}$ & $\mathbf{6}$ & $\mathbf{7}$ \\
\hline 1 & $\begin{array}{c}(400, \\
600)\end{array}$ & 500 & $\begin{array}{c}(459.8 \\
464.96)\end{array}$ & 462.38 & 0.544 & 0.0089 \\
\hline 2 & $\begin{array}{c}(400, \\
700)\end{array}$ & 550 & $\begin{array}{c}(506.32, \\
512.69)\end{array}$ & 509.5 & 0.0013 & 0.0024 \\
\hline 3 & $\begin{array}{c}(400, \\
800)\end{array}$ & 600 & $\begin{array}{c}(555.16, \\
561.03)\end{array}$ & 558.09 & 0.112 & 0.0018 \\
\hline
\end{tabular}

1: Simulation, 2: Minimum and Maximum threshold, 3: Target queue, 4: Stability range, 5: $\mathrm{q}_{0^{-}}$value, 6: $\mathrm{P}_{\max }$-value, 7 : $\beta$-value.

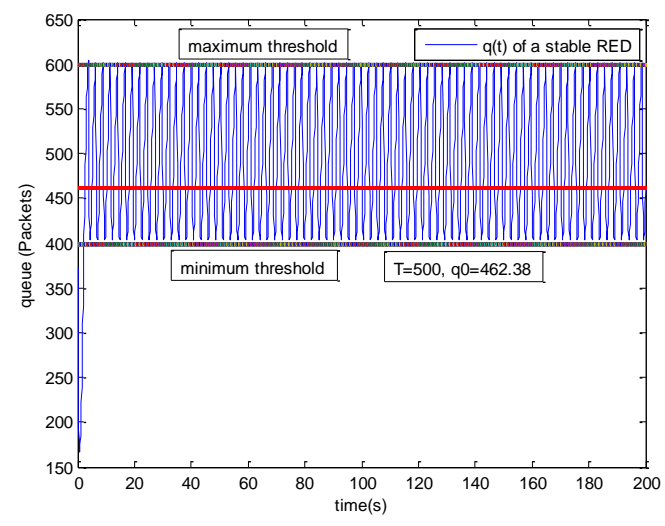

Fig.16. Queue Length when $\mathrm{T}=500$ Packets

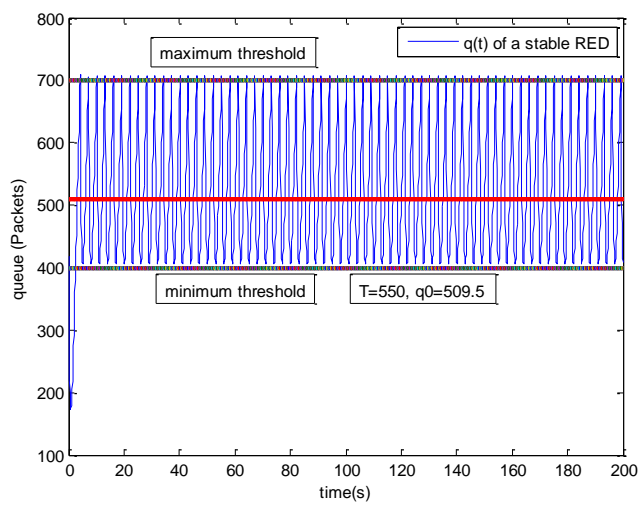

Fig.17. Queue Length when T=550 Packets

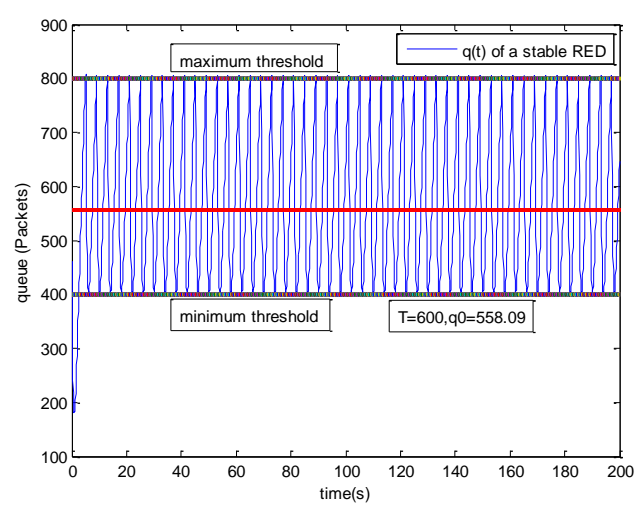

Fig.18. Queue Length when $\mathrm{T}=600$ Packets

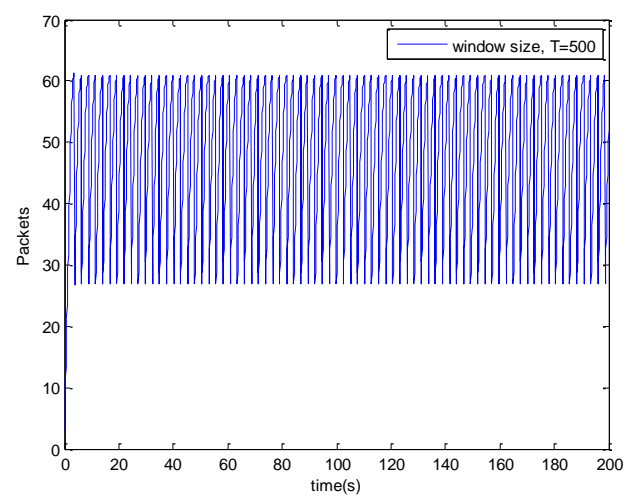

Fig.19. TCP Window when $\mathrm{T}=500$ Packets

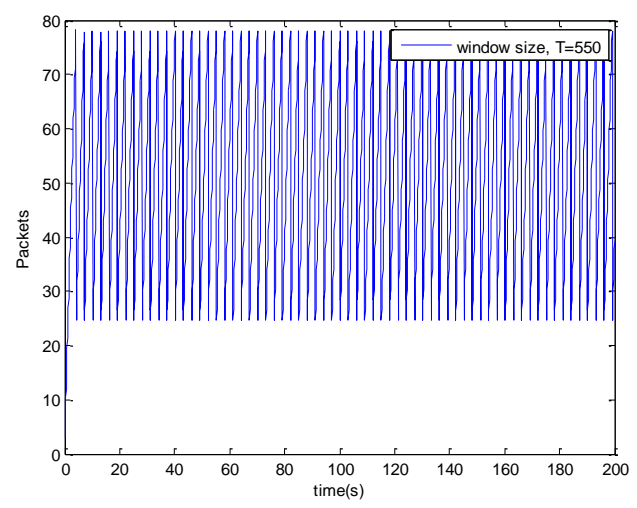

Fig.20. TCP Window when $\mathrm{T}=550$ Packets 


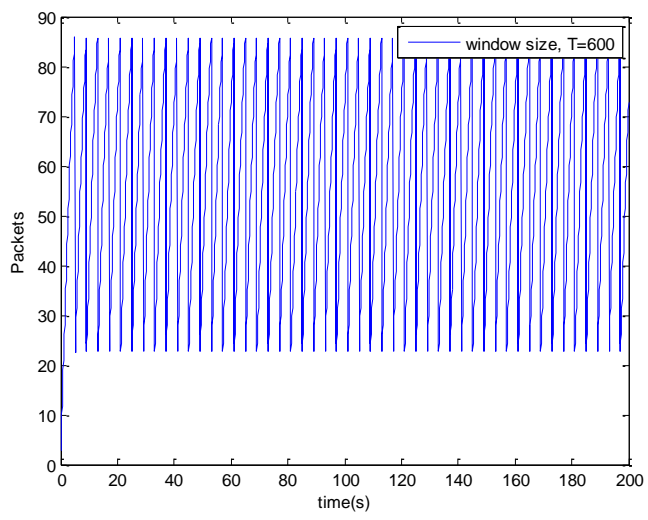

Fig.21. TCP Window when $\mathrm{T}=600$ Packets

Figs. 19, 20, and 21, illustrates the traces of TCP window dynamics for the threshold values 500, 550 and 600 respectively. Cwnd varies over the range $(26,60)$, (24, 77), and $(22,85)$ for $\mathrm{T}=500,550$, and 600 respectively. As the value of $\mathrm{T}$ increases, the lower bound of decreases whereas upper bound increases.

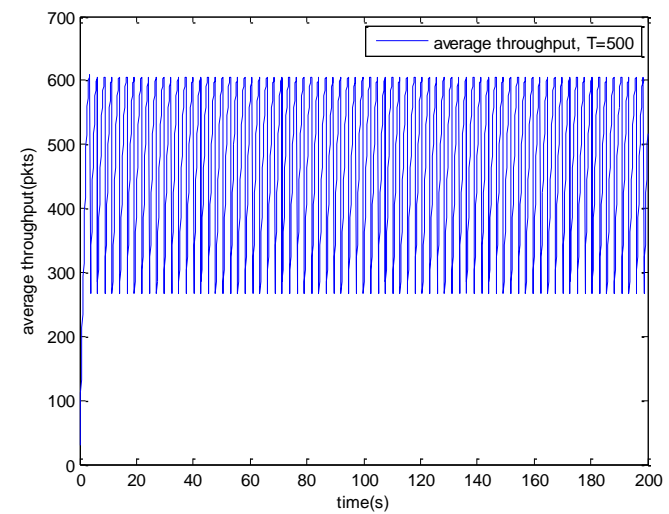

Fig.22. Average Throughput when $\mathrm{T}=500$ Packets

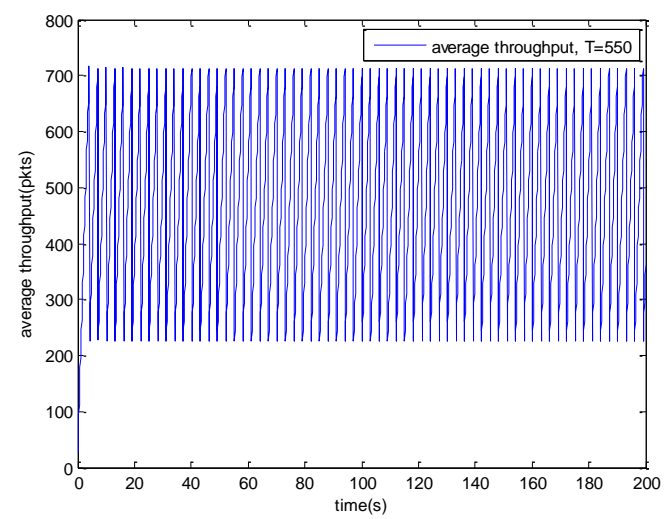

Fig.23. Average Throughput when $\mathrm{T}=550$ Packets

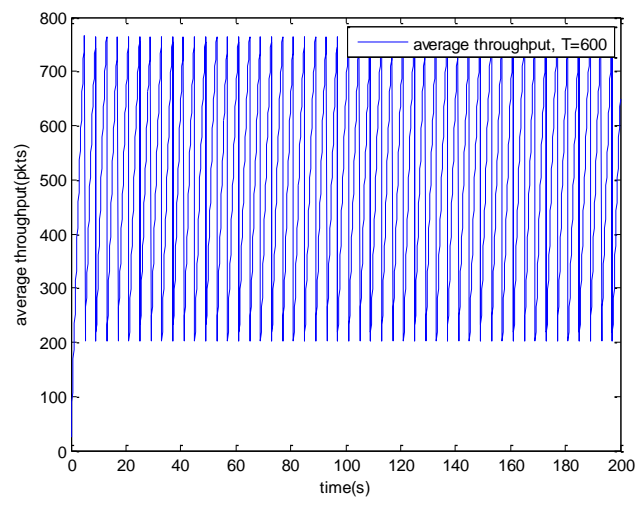

Fig.24. Average Throughput when $T=600$ Packets

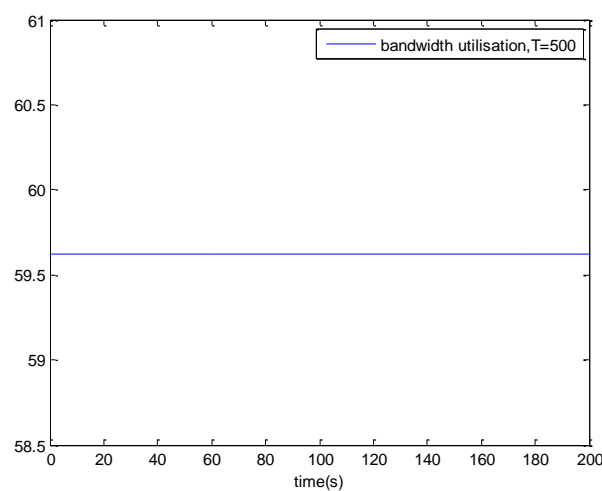

Fig.25. Bandwidth Utilisation when $\mathrm{T}=500$ Packets

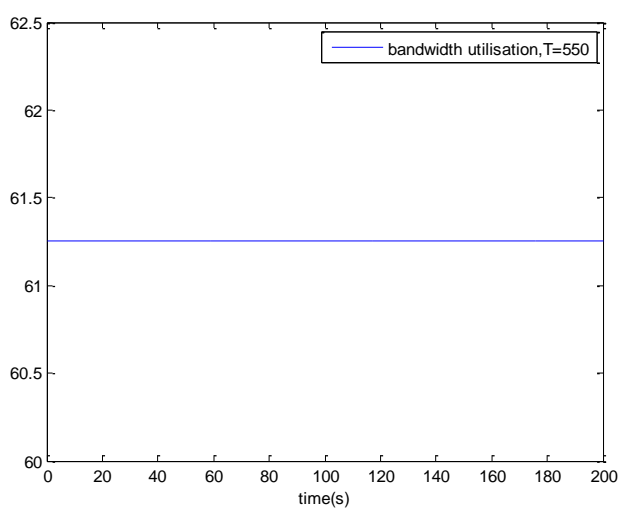

Fig.26. Bandwidth Utilisation when $\mathrm{T}=550$ Packets

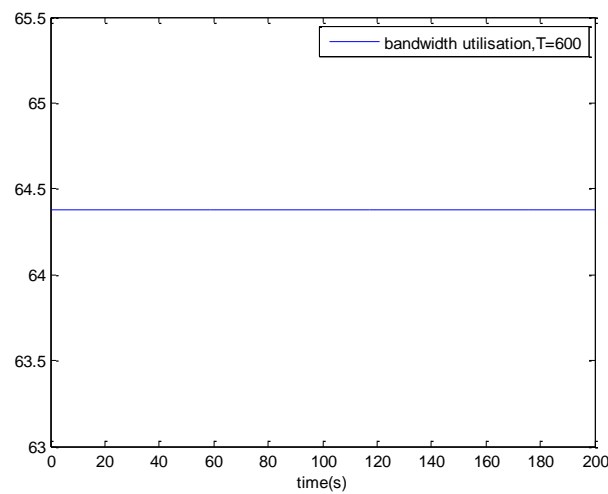

Fig.27. Bandwidth Utilisation when $\mathrm{T}=600$ Packets 
Figs. 22, 23, and 24 illustrate the traces of average throughput and Figures 25, 26, and 27 illustrates the traces of bandwidth utilisation for different threshold values. The bandwidth utilisation increases with the increase of target values.

\section{CONCLUSION}

In this paper, we enhance the performance of TCP in presence of jitter in wireless networks. Jitter is one transmission loss which occurs most frequently in wireless networks. Jitter leads to timeout loss resulting sudden drop in the throughput. The model has the capability of recognising the congestion loss from that of jitter loss. $\mathrm{k} / \mathrm{w}$ loss-predictor technique is used to achieve this task. k/w loss-predictor function uses queuing-delay and average rtt-delay (Next-RTT) as an input for prediction. When the sender TCP identified that next packet loss is due to jitter, the loss incurred in the throughput is tried to recover using immediate-recovery algorithm considering $\beta=0.1$. The proposed model also provides an end-to-end congestion control. For further improvement in the performance of the model, we discuss stability analysis. A time-delay control theory is applied. The application of asymptotic stability helps in tuning the control parameters $\mathrm{p}_{\max }$ and $\beta$ so that the network system works in a stable condition. This also controls the oscillatory behaviour of the RED router. The implementation minimises the packet loss and increases the throughput approximately by $25 \%$. In the last section of the paper, convergence analysis of queue length is discussed. An approximate solution for $\mathrm{q}(\mathrm{t})$ is derived. Convergence of $\mathrm{q}(\mathrm{t})$ for a given target value subject to that $p_{\max }$ and $\beta$ satisfies certain condition is established. The stability region of $\mathrm{q}_{0}$ is also presented there by our results provide global stability and convergence condition of the system. The described model works for networks which are associated with queuing servers with constant or variable service times. The model presented in the work is efficient, fair and adaptable to wireless networks.

\section{REFERENCES}

[1] Ivan Klimek, Marek Cajkovsky and Frantisek Jakab, "Novel methods of utilizing Jitter for Network Congestion Control", Acta Informatica Pragensia, 2(2), 124, 2013, ISSN 1805-4951.

[2] Go Hasegawa, Masashi Nakata, Hirotaka Nakano, "Receiver-based ACK splitting mechanism for TCP over wired/wireless heterogeneous networks", IEJCE Trans. Communication, Vol. E90-B, No. 5, May 2007.

[3] Saad Biaz and Nitin H.Vaidya,"Distinguishing congestion losses from wireless transmission losses: A negative result", Department of Computer Science, Texas A and M University, TX, 77843-3112, USA.

[4] Saad Biaz, and Nitin H.Vaidya, "Sender-based heuristics for distinguishing congestion losses from wireless transmission losses", Department of Computer Science, Texas Aand M University, USA, 2003.

[5] Toni Janevski, and Strahil Panev, "Modifications for TCP improvements over wireless networks", 18th
Telecommunications forum TELFOR-2010, Serbia, Belgrade, November 2010.

[6] Geoff Huston, "TCP in a wireless world", IEEE Internet computing, 1089- 7801/01, April 2001

[7] H. Balakrishnan, S. Seshan, E. Amir and R. H. katz., "Improving TCP/IP Performance Over Wireless Networks," In Proceedings of ACM MOBICOM '95, 1995.

[8] A. Bakre and B. R. Badrinath, "I-TCP: Indirect TCP for Mobile Hosts," In Proceedings of 15th International Conf. on Distributed Computing Systems (ICDCS), May 1995.

[9] H. Balakrishnan, S. Seshan, E. Amir and R. H. katz., "Improving TCP/IP Performance over wireless networks," In Proceedings of ACM MOBICOM '95, 1995.

[10] P. Sinha et al., "WTCP: A Reliable Transport Protocol for Wireless Wide-Area Networks," In Proceedings of ACM MOBICOM 1999, Aug. 1999.

[11] S. Mascolo and C. Casetti, "TCP Westwood: Bandwidth Estimation for Enhanced Transport over Wireless Links," In Proceedings of MOBICOM '2001.

[12] Seongho Cho, and Heekyoung Woo, "TCP Performance Improvement with ACK Pacing in Wireless Data Networks", School of Electrical Engineering and Computer Science, Seoul National University, Seoul, Republic of Korea.2005.

[13] Masashi Nakata, Go Hasegawa, Hirotaka Nakano, "Modeling TCP throughput over wired/wireless heterogeneous networks for receiver-based splitting mechanism", Graduate school of Information Science and Technology, Osaka University, Japan.

[14] Eric Hsiao-Kuang Wu, and Mei-Zhen Chen," JTCP: Jitter-based TCP for heterogeneous wireless networks", IEEE journal on selected areas in communications, Vol. 22, No. 4, May 2004.

[15] H. Balakrishnan and R. H. Katz, "Explicit Loss Notification and Wireless Web Performance," In Proceedings of IEEE GLOBECOM '98, 1998.

[16] L.Tan, W.Zhang, G.Peng and G.Chen, "Stability of TCP/RED systems in AQM routers", IEEE Trans. Automatic control, Vol. 51, No. 8, pp 1393-1398, August 2006.

[17] W.Zhang. L.Tan, and G.Peng, "Dynamic queue level control of TCP/RED systems in AQM routers", Computers and Electrical Engineering, Vol.35, No. 1, PP 59-70, 2009.

[18] Hiroaki Mukaidani, Lin Cai, Xuemin Shen, "Stable queue management for supporting TCP flows over wireless networks", IEEE ICC, 978-1-61284-231-8/11, 2011.

[19] S. Goankar, R.R.Choudhury and Robin Kravets, "Designing a rate-based transport protocol for wiredwireless networks", Department of Computer Science, University of Illinois, Urbana Champaign, IL.

[20] Shankaraiah and Pallapa Venkataram, "Transaction-based QoS management in a hybrid wireless superstore environment", I.J. Computer Network and Information Security, 2, 1-11, Published Online March 2011 in MECS

[21] Adira Quintero, Francisco Sans, and Eric Gamess," Performance Evaluation of IPv4/IPv6 Transition Mechanisms", I. J. Computer Network and Information Security, 2, 1-14 Published Online February 2016 in MECS. 


\section{Authors' Profiles}

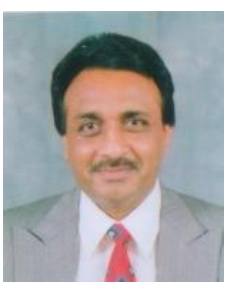

N.G.Goudru received his Ph.D degree in Mathematics from Gulbarga University, Karnataka in 1989, M.Sc (Applied Mathematics) degree from Karnataka University, Dharwar in 1981, M.S (Software Systems) degree from BITS, Pilani in 1994. He is working as a professor in the department of Mathematics, BMS Institute of Technology, Bangalore, Karnataka. His major areas of research are in Computational fluid dynamics and wireless mobile networks.

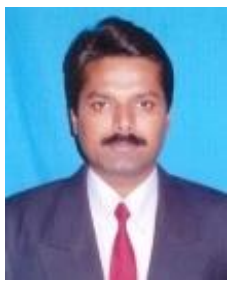

B.P.Vijaya Kumar received his $\mathrm{Ph}$. D degree in Electrical and Communication Engineering. department from Indian Institute of Science (IISc), Bangalore in 2003, M.Tech degree in Computer Science and Technology from the University of Roorkee (Presently Indian Institute of Technology, IITR), with honors in 1992 and Bacholers degree in Electronics and Communications from Mysore University with Distinction in the year 1987. He is currently working as a professor and Head of Information Science and Engineering department, MS Ramaiah Institute of Technology, Bangalore, Karnataka, India, where he is involved in research and teaching UG and PG students, and his major area of research are Computational Intelligence applications in Mobile, Adhoc and Sensor networks.

How to cite this paper: Goudru N.G, Vijaya Kumar B.P,"Performance Analysis and Enhancement of TCP in Presence of Jitter in Wireless Networks", International Journal of Computer Network and Information Security(IJCNIS), Vol.8, No.6, pp.9-21, 2016.DOI: 10.5815/ijcnis.2016.06.02 